University of Nebraska - Lincoln

DigitalCommons@University of Nebraska - Lincoln

2009

\title{
Development of a Source of Quasi-Monochromatic MeV Energy Photons
}

Donald Umstadter

University of Nebraska - Lincoln, donald.umstadter@unl.edu

Sudeep Banerjee

University of Nebraska-Lincoln, Sudeep.Banerjee@asu.edu

Viswanathan Ramanathan

University of Nebraska - Lincoln, ramanath@cse.unl.edu

Nathan D. Powers

University of Nebraska-Lincoln, ndp5@byu.edu

Nathaniel Cunningham

University of Nebraska - Lincoln, ncunning@nebrwesleyan.edu

See next page for additional authors

Follow this and additional works at: https://digitalcommons.unl.edu/physicsumstadter

Part of the Physics Commons

Umstadter, Donald; Banerjee, Sudeep; Ramanathan, Viswanathan; Powers, Nathan D.; Cunningham, Nathaniel; and Chandler-Smith, Nathan A., "Development of a Source of Quasi-Monochromatic MeV Energy Photons" (2009). Donald Umstadter Publications. 78.

https://digitalcommons.unl.edu/physicsumstadter/78

This Article is brought to you for free and open access by the Research Papers in Physics and Astronomy at DigitalCommons@University of Nebraska - Lincoln. It has been accepted for inclusion in Donald Umstadter Publications by an authorized administrator of DigitalCommons@University of Nebraska - Lincoln. 


\section{Authors}

Donald Umstadter, Sudeep Banerjee, Viswanathan Ramanathan, Nathan D. Powers, Nathaniel Cunningham, and Nathan A. Chandler-Smith 


\title{
Development of a Source of Quasi-Monochromatic MeV Energy Photons
}

\author{
Donald Umstadter, Sudeep Banerjee, Vidya Ramanathan, Nathan Powers, \\ Nathaniel Cunningham, and Nate Chandler-Smith
}

Department of Physics and Astronomy, University of Nebraska, Lincoln

\begin{abstract}
We report current progress on a project to develop an all-optically-driven x-ray photon source. A laser pulse with 40-50 TW of peak power is focused on a supersonic helium nozzle to drive a relativistic plasma wave. Electron beams with energies of $320 \mathrm{MeV}(+/-28 \mathrm{MeV})$ are accelerated by means of laser wakefield acceleration. Remarkably, the acceleration region is only $3 \mathrm{~mm}$ in length. This accelerator is currently being employed to demonstrate the generation of MeV-energy x-ray by means of all-optical Thomson scattering. By this mechanism, a lower power, laser pulse (from the same laser system) is focused onto the above laser-driven electron beam, 1-eV energy photons are Doppler-shifted in energy to $>1 \mathrm{MeV}$
\end{abstract}

Keywords: x-rays, Thomson scattering, wakefield acceleration

PACS: 07.77.Ka; 52.40.Db; 07.85.Fv

\section{INTRODUCTION}

Thomson scattering of intense laser light from a relativistic electron beam is a promising route to the development of a compact mono-energetic x-ray source, which promises to significantly reduce the dose and scattered radiation background, as compared with Bremsstrahlung sources. These features are important in applications such as active interrogation of nuclear materials, remote sensing, and nondestructive testing. In Thomson scattering from an energetic electron beam $(300 \mathrm{MeV})$, a $1-\mathrm{eV}$ energy laser light can be Doppler-shifted to $1.5 \mathrm{MeV}$ energy, scaling as $4 \gamma^{2}$, where $\gamma$ is the relativistic factor associated with the electron beam energy. ${ }^{1,2}$ The $x-$ ray beam is also highly collimated (mrad). Also, because the pulse duration can be ultrashort (fs), Thomson x-rays can be used to study of ultrafast chemical, biological, and physical processes, such as inner-shell electronic or phase transitions.

The physics of Thomson scattering is comparable to $x$-ray generation by a fixed magnet undulator, as in a synchrotron, but with several important differences/advantages. These principally arise from the much shorter wavelength of a high-frequency (optical) electromagnetic wiggler (micron vs. cm). First, a much shorter wiggler section ( $\sim \mathrm{mm}$ vs. $\sim 10 \mathrm{~m})$ is required. Second, a much lower energy for the electron beam $(\sim 100 \mathrm{MeV}$ vs. $\sim 10 \mathrm{GeV})$ is required.
Last, much higher maximum photon energy $(\sim \mathrm{MeV}$ vs. $100 \mathrm{keV}$ ) can be produced.

If, additionally, the electron beam is accelerated by the ultra-high gradient wakefield plasma wave, ${ }^{3}$ driven by a light pulse from the same laser system that produces the scattering pulse, then the combined accelerator and wiggler regions would be only a few millimeters in length. ${ }^{4}$ Figure 1 shows a schematic diagram of $x$-ray generation by means of this alloptically-driven Thomson scattering.

\section{ROUTE TO MONOENERGETIC X- RAYS}

At the newly established Extreme Light Laboratory, all of the components required to build this device, including the laser system (delivering peak power $>100 \mathrm{TW}$ at a repetition rate of $10 \mathrm{~Hz}$ ), and electron accelerator (delivering beams with energy up to $>300 \mathrm{MeV}$ and divergence of 5-6 mrad), have now been developed and fully characterized, and are discussed in detail below.

\section{Diocles High-Peak-Power Laser System}

Construction of a high-power laser system (Diocles) was recently completed. It has demonstrated unprecedented levels of performance for a petawatt- 
class system-with respect to beam quality, stability, and reproducibility. Also, the implementation of a deformable mirror enabled near-diffraction limited focusing. The measured laser parameters are shown in Table 1, and a schematic diagram of the system is shown in Fig. 1.

TABLE 1. Measured Laser Parameters

\begin{tabular}{|l|l|}
\hline Laser Parameter & Measurement \\
\hline Peak power & $140 \mathrm{TW}$ \\
\hline Repetition rate & $10 \mathrm{~Hz}(0.1 \mathrm{~Hz})$ \\
\hline Central wavelength & $805 \mathrm{~nm}$ \\
\hline Pulse duration & $<30 \mathrm{fs}$ \\
\hline Pulse energy & $3.5 \mathrm{~J}($ compressed) \\
\hline Energy stability & $0.8 \% \mathrm{rms}, 4.9 \%$ \\
\hline Strehl ratio & 0.95 \\
\hline Pointing stability & $(1 \mathrm{~min}): 3.5 \mu \mathrm{rad}$ \\
\hline
\end{tabular}

\section{Laser wakefield electron accelerator}

When a pulse from the Diocles laser was focused on a supersonic helium nozzle, a relativistic plasma wave was driven to large amplitude, and an electron beam was accelerated to high energy. The dependence of the accelerator parameters on laser and plasma parameters was determined. The optimal accelerator operating points were also found. Progress had been made recently in the acceleration of monoenergetic beams by operating in the so-called "bubble" or "forced-wakefield" regime. 5 Further progress was made by simultaneously matching several of the laser and plasma parameters. In our experiment, we demonstrated that the highest quality electron beams are produced when all of the laser and plasma parameters are nearly "matched." In this case, the laser pulse does not suffer from the effects of nonlinear pulse evolution because it initially begins the interaction with its pulse duration and focal spotsize in resonance with the plasma. This mitigates the instability and irreproducibility associated with mismatched parameters, characteristic of the earliest wakefield accelerator experiments. ${ }^{7,8}$ In order to satisfy the constraints required of matching, the laser parameters used were $40-\mathrm{TW}$ peak power, and pulse duration of $30 \mathrm{fs}$. The plasma density was chosen to be resonant with the laser. The resulting electron beam charge was $600 \mathrm{pC}$; its brightness is $3.0 \times 10^{8}$ electrons $\mathrm{mrad}^{-2}$, and its fluctuation level was less than $1 \%$ in pointing and energy. The electron beam energy was $>$ $300 \mathrm{MeV}$. A typical spectrum, in which the beam has energy of $320 \mathrm{MeV}$, an energy spread of $10 \%$ (limited by the spectrometer resolution), and an angular divergence of $6 \mathrm{mrad}$, is shown in Fig. 3. Here, the $x-$ axis corresponds to the energy, and the $y$-axis to pointing and angular spread of the beam. Note the lack of any visible dark current at lower laser energies. Some of these accelerator parameters are summarized in Table 2.

The stability and reproducibility of the accelerator was also investigated in detail, and was found to sensitively depend on the laser contrast. Preliminary experiments in a low-laser-contrast mode, corresponding to a nanosecond pedestal of $3 \times 10^{-7}$, produced electron beams with large shot-to-shot angular variation of (10 mrad), and large energy fluctuation (approximately 20-30\% shot-to-shot). When the contrast of the laser system was improved to $2 \times 10^{-8}$, the stability of the electron accelerator also improved. Some of these stability results are summarized in Table 3 . These technological improvements permits the use of these electron accelerators for numerous applications, including nuclear activation analysis--over large standoff distances, as well as the production of quasimonoenergetic hard x-rays, radiography, discussed below.

\section{All-Optically-Driven Thomson Scattering X-Ray Photon Source}

Proof-of-principle experiments are currently underway to use the electron beam and laser pulse discussed above to generate $1-\mathrm{MeV}$ energy $\mathrm{x}$-rays by means of Thomson scattering. The predicted spectral distribution of $x$-ray photons generated is $\Delta \omega / \omega(\theta=0)$ equals $11 \%$. The $\mathrm{x}$-ray photon beam is predicted to be well collimated, with up to $2.6 \times 10^{8}$ photons per laser shot, assuming the experimentally measured charge of 600 pC. The Diocles laser system will soon be upgraded in peak power by an order of magnitude, to the PW level. This power upgrade will permit access to the highly relativistic Thomson scattering regime, as well as the use of higher energy scattering pulses of arbitrary duration. 

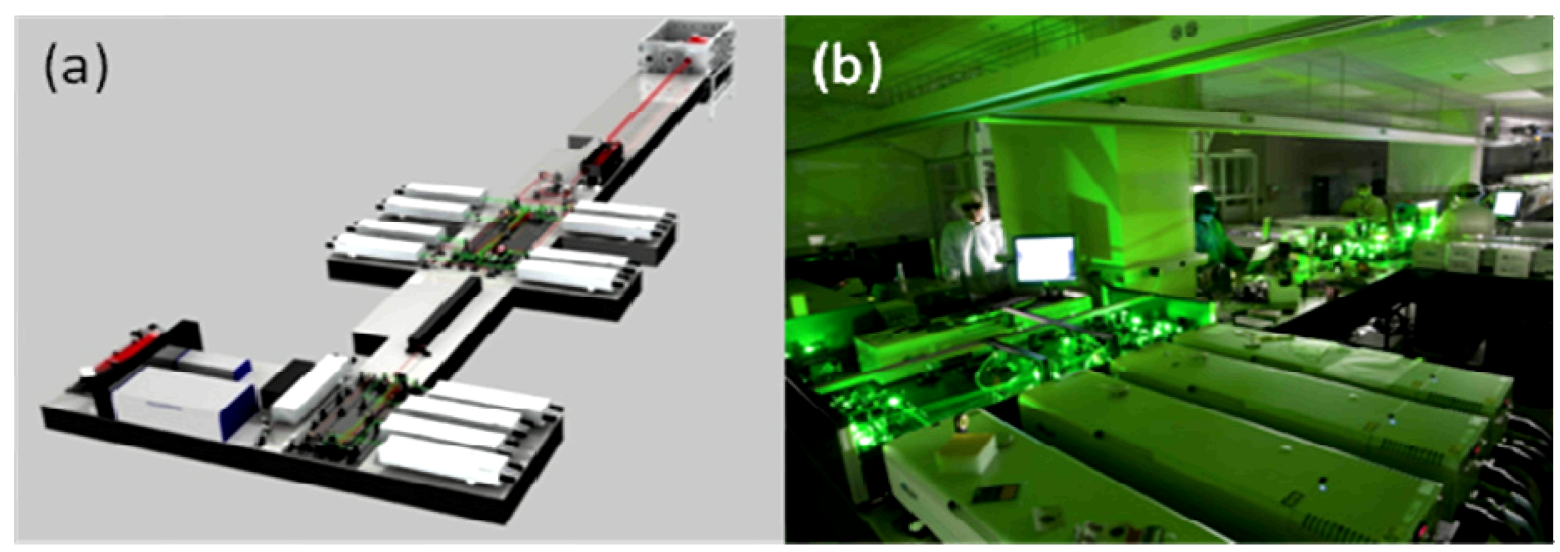

FIGURE 1. (a) Schematic diagram and (b) photo of the Diocles laser system.

TABLE 2. Measured Parameters of the UNL Laser-Driven Wakefield Accelerator

\begin{tabular}{|l|l|}
\hline Electron accelerator parameters & Measured results \\
\hline Drive laser peak power & $45 \mathrm{TW}$ \\
\hline Electron energy & $320 \mathrm{MeV}$ \\
\hline$\square \mathrm{E} / \mathrm{E}$ & $10 \%$ \\
\hline Charge per bunch & $600 \mathrm{pC}$ \\
\hline Electron beam divergence & $6 \mathrm{mrad}$ \\
\hline Pointing and energy stability & $1 \%$ over 30 shots \\
\hline
\end{tabular}

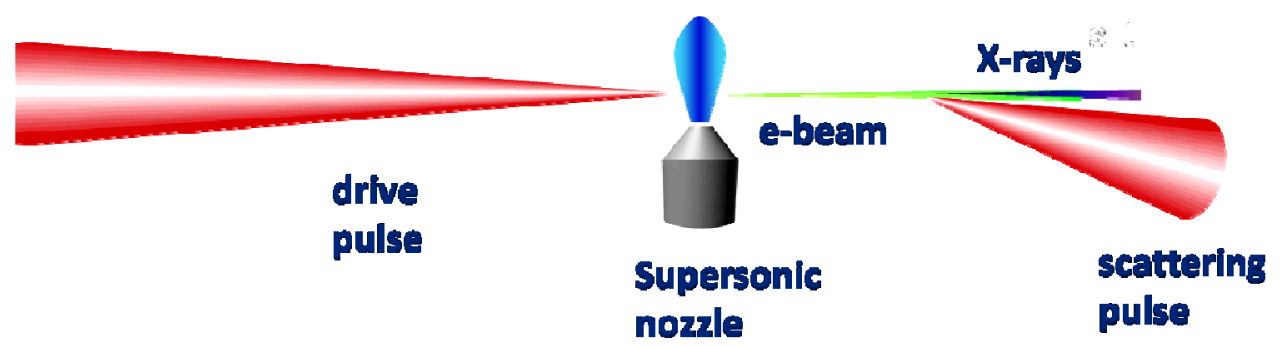

FIGURE 3. Schematic diagram of x-ray generation by all-optically-driven Thomson scattering

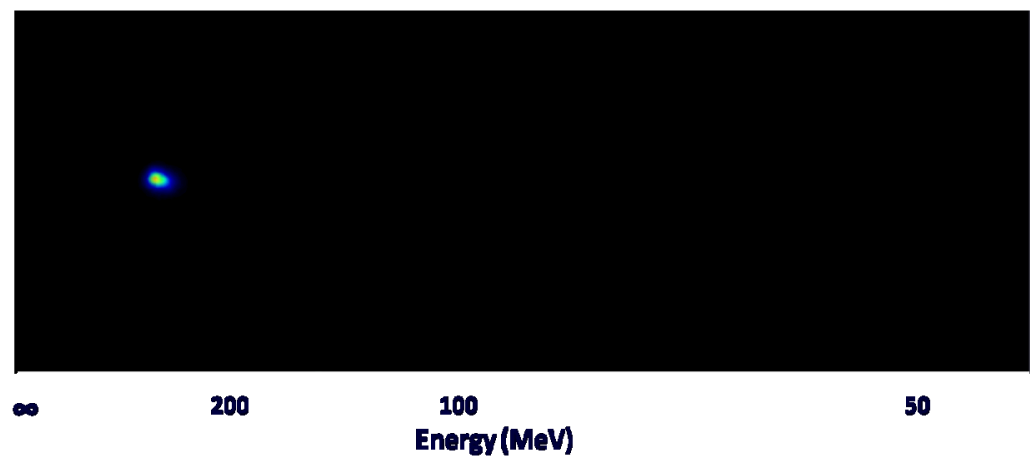

FIGURE 2. Monoenergetic, low-divergence beam, generated by 45 TW of laser power focused into a plasma with density of $7 \times 10^{18} \mathrm{~cm}^{-3}$. The energy is peaked around $320 \mathrm{MeV}$ with a spread of $10 \%$. The angular divergence of the beam (vertical axis) is $6 \mathrm{mrad}$. 
TABLE 3. Stability of the UNL Electron Accelerator

\begin{tabular}{|l|l|l|l|l|}
\hline Electron beam parameter & $\begin{array}{l}\text { Angular position } \\
(\mathrm{mrad})\end{array}$ & $\begin{array}{l}\text { Divergence } \\
(\mathrm{mrad})\end{array}$ & $\begin{array}{l}\text { Energy } \\
(\mathrm{MeV})\end{array}$ & $\begin{array}{l}\text { Energy spread } \\
(\mathrm{MeV})\end{array}$ \\
\hline Mean & 0 & 5.3 & 344 & 38.4 \\
\hline Standard deviation & 1.1 & 1.7 & 35 & 4.8 \\
\hline
\end{tabular}

\section{ACKNOWLEDGEMENTS}

The authors gratefully acknowledge the support of Domestic Nuclear Detection Office of the Department of Homeland Security, Defense Advanced Projects Agency, Air Force Office of Scientific Research, and Chemical Sciences, Biosciences, and Geosciences Division in the Office of Basic Energy Sciences of the Department of Energy.

\section{REFERENCES}

1. Y.Y. Lau, F. He and D. Umstadter, Phys. Plasmas 10 , 2155 (2003); F. He, Y. Y. Lau, D. P. Umstadter, and R. Kowalczyk, Phys. Rev. Lett., 90, 055002 (2003).

2. F He, YY Lau, DP Umstadter, T Strickler, Phys. Plasmas, 9 (10): 4325-4329 (2002) ; F. He YY Lau, D. Umstadter, T. Strickler, Phys. Plasmas 10, 327 (2003).
3. T. Tajima and J. Dawson, Phys. Rev. Lett., 43, 267 (1979).

4. S. Y. Chen, A. Maksimchuk and D. Umstadter, Nature, 396, 653 (1998); S. Banerjee, A. R. Valenzuela, R. C. Shah, A. Maksimchuk, D. Umstadter, J. Mod. Optics 49, 2599(2002); D. Umstadter, "Laser-driven x-ray sources," in 2003 Yearbook in Science and Technology, New York, McGraw-Hill, 2003, p. 215; K.T. Phuoc, A. Rousse, M. Pittman, J.P. Rousseau, V. Malka, S. Fritzler, D. Umstadter, and D. Hulin, Phys. Rev. Lett., 91, 195001-1 (2003).

5. A. Pukhov and J. Meyer-Ter-Ven, Appl. Phys. B 74, 355 (2002); J. Faure et al., Nature 431, 541 (2004); S. Mangles et al.," Nature 431, 535 (2004); C. Geddes et al., Nature 431, 538 (2004).

6. S. P. D. Mangles et al., Phys. Plasmas 14, 056702 (2007).

7. A. Modena et al. Nature. 377, 606 (1995).

8. D. Umstadter, S.-Y. Chen, A. Maksimchuk, G. Mourou and R. Wagner, Science 273, 472 (1996) 